This is the author's final, peer-reviewed manuscript as accepted for publication. The publisher-formatted version may be available through the publisher's web site or your institution's library.

\title{
Identification and characterization of a novel chitinase-like gene cluster (AgCht5) possibly derived from tandem duplications in the African malaria mosquito, Anopheles gambiae
}

Jianzhen Zhang, Xin Zhang, Yasuyuki Arakane, Subbaratnam Muthukrishnan, Karl J. Kramer, Enbo Ma, Kun Yan Zhu

\section{How to cite this manuscript}

If you make reference to this version of the manuscript, use the following information:

Zhang, J., Zhang, X., Arakane, Y., Muthukrishnan, S., Ma, E., Kramer, K.J., Zhu, K.Y. (2011). Identification and characterization of a novel chitinase-like gene cluster (AgCht5) possibly derived from tandem duplications in the African malaria mosquito, Anopheles gambiae. Retrieved from http://krex.ksu.edu

\section{Published Version Information}

Citation: Zhang, J., Zhang, X., Arakane, Y., Muthukrishnan, S., Ma, E., Kramer, K.J., Zhu, K.Y. (2011). Identification and characterization of a novel chitinase-like gene cluster (AgCht5) possibly derived from tandem duplications in the African malaria mosquito, Anopheles gambiae. Insect Biochemistry and Molecular Biology, 41(8), 521528.

Copyright: Copyright @ 2011, Elsevier

Digital Object Identifier (DOI): doi:10.1016/j.ibmb.2011.03.001

Publisher's Link: www.elsevier.com/locate/ibmb

This item was retrieved from the K-State Research Exchange (K-REx), the institutional repository of Kansas State University. K-REx is available at http://krex.ksu.edu 
Identification and characterization of a novel chitinase-like gene cluster

11 Jianzhen Zhang ${ }^{\mathrm{a}, \mathrm{b}}$, Xin Zhang ${ }^{\mathrm{b}}$, Yasuyuki Arakane ${ }^{\mathrm{c}, \mathrm{d}}$, Subbaratnam Muthukrishnan ${ }^{\mathrm{c}}$, Karl J.

12 Kramer $^{\mathrm{c}}$, Enbo Ma ${ }^{\mathrm{a}}$ and Kun Yan Zhu ${ }^{\mathrm{b}, *}$

${ }^{a}$ Research Institute of Applied Biology, Shanxi University, Taiyuan, Shanxi 030006, China

${ }^{\mathrm{b}}$ Department of Entomology, 123 Waters Hall, Kansas State University, Manhattan, KS 66506, USA

${ }^{c}$ Department of Biochemistry, 141 Chalmers Hall, Kansas State University, Manhattan KS 66506, USA

${ }^{\mathrm{d}}$ Division of Plant Biotechnology, College of Agriculture and Life Science, Chonnam National University, Gwangju 500-757, Korea

* Corresponding author: Tel.: +1 785532 4721; fax: +1 7855326232. 
Insect chitinase 5 (Cht5), a well-characterized enzyme found in the molting fluid and/or

30 integument, is classified as a group I chitinase and is usually encoded by a single gene. In this study, a Cht5 gene cluster consisting of five different chitinase-like genes (AgCht5-1, AgCht5-2, AgCht5-3, AgCht5-4 and AgCht5-5) was identified by a bioinformatics search of the genome of Anopheles gambiae. The gene models were confirmed by cloning and sequencing of the corresponding cDNAs and gene expression profiles during insect development were determined. All of these genes are found in a single cluster on chromosome 2R. Their open reading frames (ORF) range from 1227 to 1713 bp capable of encoding putative proteins ranging in size from 409 to 571 amino acids. The identities of their cDNA sequences range from 52 to $66 \%$, and the identities of their deduced amino acid sequences range from 38 to $53 \%$. There are four introns for $A g C h t 5-1$, two for $A g C h t 5-2$ and $A g C h t 5-3$, only one for $A g C h t 5-4$, but none for $A g C h t 5-5$ in the genome. All five chitinase-like proteins possess a catalytic domain with all of the conserved sequence motifs, but only AgCht5-1 has a chitin-binding domain. Phylogenetic analysis of these deduced proteins along with those from other insect species suggests that AgCht5-1 is orthologous to the Cht5 proteins identified in other insect species. The differences in expression patterns of these genes at different developmental stages further support that these genes may have distinct functions. Additional searching of the genomes of two other mosquito species led to the discovery of four Cht5-like genes in Aedes aegypti and three in Culex quinquefasciatus.

48 Thus, the presence of a Cht5 gene cluster appears to be unique to mosquito species and these genes may have resulted from gene tandem duplications. 
50 Keywords: African malaria mosquito, Anopheles gambiae, Chitinase, Gene cluster, Gene

51 duplication, Glycoside hydrolase

52

53

54

55

56

57

58

59

60

61

62

63

64

65

66

67

68

69

70

71 


\section{Introduction}

Chitinases (EC.3.2.1.14) are enzymes responsible for hydrolyzing glycosidic bonds in chitin and widely distributed in nature, including vertebrates, microorganism and even plants. Mammals are not known to synthesize chitin or metabolize chitin as a nutrient, yet the human genome encodes eight different chitinases of the glycoside hydrolase 18 (GH18) family, which play an important role in T-cell mediated inflammation and asthma (Funkhouser et al., 2007; Reese et al., 2007; Shuhui et al., 2009).

In insects, chitin associates with proteins to form the cuticular exoskeleton and peritrophic matrix (PM) in the midgut lumen. During a molting cycle, a part of the old cuticle is digested while new chitin is synthesized and deposited (Reynolds et al., 1996). It has been suggested that insect chitinases may have multiple functions including defense, digestion and molting (Shen and Jacob-Lorena, 1997; Filho et al., 2002; Zheng et al., 2002; Genta et al., 2006; Zhu et al., 2008b). Indeed, a chitinase expressed in the gut of European corn borer (Ostrinia nubilalis) has been identified and it has been proposed that this enzyme is responsible for regulation of chitin content of PM and growth of $O$. nubilalis larvae (Khajuria et al., 2010). A chitinase was also purified from the venom gland of an endoparasitic wasp Chelonus sp. near curuimaculatus (Krishnan et al., 1994). Because of the crucial roles of chitinases in insect growth and development, these enzymes have been widely recognized as potential targets for developing chemical pesticides for insect control (Royer et al., 2002; Hirose et al., 2010). Insect chitinases belong to the GH18 multi-gene family with a rapid increase in the number of genes identified as the annotation of completed genome sequences of several 
insect species has occurred (Zhu et al., 2004, 2008a). At present, insect chitinases and chitinase-like proteins are classified into eight groups based on a phylogenetic analysis of their catalytic domains (Arakane and Muthukrishnan, 2010). Among these chitinases and chitinase-like proteins, chitinase 5 (Cht5) is classified into group I.

To date, only a single $C h t 5$ gene has been identified from each of several insect genomes and the representatives of this gene have been well characterized in several lepidopteran and coleopteran species (Kim et al.,1998; Shinoda et al., 2001; Zheng et al., 2002; Ahmad et al., 2003; Fitches et al., 2004; Bolognesi et al., 2005; Zhu et al., 2008a). All insect Cht5s have a typical multidomain structural organization that includes a signal peptide, a catalytic domain, a PEST-like linker region enriched in proline $(\mathrm{P})$, glutamic acid $(\mathrm{E})$, serine (S) and threonine (T) that is heavily glycosylated, and a cysteine-rich chitin-binding domain. The sizes of Cht5 enzymes range from 552 to 586 amino acid residues. The transcripts of $C h t 5$ are mainly detected in the epidermis and the gut, and its expression increases during the molting process (Royer et al., 2002). In Helicoverpa armigera, Cht5 is also expressed in the fatbodies (Ahmad et al., 2003). However, the expression of Cht5 has not been reported in haemocytes from any insect species. In Manduca sexta, the transcript level of $C h t 5$ can be induced by 20-hydroxyecdysone, but it is suppressed by the juvenile hormone analog, fenoxycarb (Kramer et al., 1993). The recombinant protein expressed in an insect cell line showed high levels of chitinolytic activity (Gopalakrishnan et al., 1995; Zheng et al., 2003; Ahmad et al., 2003).

It is likely that Cht5 may be involved in chitin turnover associated with molting. In Tribolium castaneum, RNA interference was performed to silence $T c C h t 5$. The insects that 
116 were injected with double-stranded RNA (dsRNA) for TcCht5 exhibited a lethal phenotype

117 only at the pharate adult stage. At the time of death, some of the adult cuticle was visible

118 under the old pupal cuticle which was not shed, suggesting that $T c C h t 5$ is required for

119 pupal-adult molting (Zhu et al., 2008b). All these results indicate that Cht5 is an essential

120 enzyme for insect growth and development.

121 Although insects have been known to have only a single Cht5 gene, our recent studies

122 have revealed a novel Cht5 gene cluster consisting of multiple chitinase-like genes in three

123 mosquito species. In this paper, we report: 1) identification of a cluster of five An. gambiae

124 Cht5-like genes (AgCht5-1, AgCht5-2, AgCht5-3, AgCht5-4 and AgCht5-5) and their

125 chromosomal localization, 2) characterizations of their gene models and developmental

126 expression patterns, and 3) the results of a comparative investigation on $C h t 5$ gene clusters in

127 two other mosquito species including Aedes aegypti and Culex quinquefasciatus. This is the

128 first demonstration of gene duplication of this group of chitinase genes, which may be unique

129 to the mosquito lineage.

\section{2. Materials and Methods}

131

132

133

134

135

136

\subsection{Insect culture}

An. gambiae was obtained from the Malaria Research and Reference Reagent Resource Center (MR4, Manassas, VA) and has been cultured in the Department of Entomology at Kansas State University, Manhattan, KS since 2005. The colony was maintained based on the methods previously described (Zhang and Zhu, 2006)

\subsection{Sequencing of cDNAs of chitinase 5 gene cluster}


chitinase-like genes in the genome of An. gambiae. Based on the bioinformatics analysis, we

139 identified a chitinase 5-like gene (AgCht5) (accession no: XP_001237469.2) that has been

140 annotated as one encoding a large protein with five chitinase catalytic domains. Each

141 chitinase-coding domain of this gene was then searched against the An. gambiae EST

142 database. Individual EST clones obtained from the MR4 were sequenced. For the EST clones

143 missing the 3'-end sequences of $A g C h t 5-1$ and $A g C h t 5-4,3$ '-RACE PCR was performed to

144 obtain their full-length cDNA by using the $\mathrm{SMART}^{\mathrm{TM}}$ RACE cDNA Amplification Kit

145 (Clontech, Mountain View, CA). The primer sequences for 3'-RACE PCR are shown in

146 Table 1. The PCR products were subcloned into the TA cloning vector (Invitrogen, Carlsbad,

147 CA) according to the manufacturer's protocol and sequenced by DNA Sequencing Facility at 148 Kansas State University (Manhattan, KS).

149 Multiple sequence alignment was performed using NPS@ (Network Protein Sequence

150 Analysis). Identity comparisons among the five AgCht5 sequences were performed by using

151 DNAstar (Madison, WI). SMART domain analysis (http://smart.embl-heidelberg.de/; Schultz

152 et al., 1998) and UCSC genome bioinformatics programs (http://genome.ucsc.edu) were used

153 to predict the domain architecture and gene structure of each identified chitinase and

154 chitinase-like gene, respectively. The phylogenetic tree was constructed based on the amino

155 acid sequences of their catalytic domains by the neighbor-joining algorithm using Mega 4.0

156 software (Tamura et al., 2007).

157 2.3. Analysis of developmental stage-dependent gene expression patterns 

including eggs; first-, second-, third- and fourth-instar larvae; and adults of An. gambiae were

160 evaluated in the study. For more detailed developmental expression patterns, mosquito eggs

161 and pupae were collected at several time points for each developmental stage. Total RNA

162 was isolated using the Trizol reagent (Invitrogen) and treated with DNase I (Fermentas, Glen

163 Burnie, MD). The first-strand cDNA was synthesized using a First Strand cDNA Synthesis

164 Kit (Fermentas) according to the manufacturer's instructions. Beacon 7.0 software was used

165 for primer design and ribosomal protein S3 (Rps3) was used as an internal reference gene.

166 The primers used for expression analysis are shown in Table 1. RT-PCR was carried out in a

$167 \quad 25-\mu 1$ reaction mixture containing $1 \mu \mathrm{l}$ template cDNA, $12.5 \mu \mathrm{l}$ Taq Master Mix (Fermentas),

$168 \quad 0.2 \mu \mathrm{M}$ of each primer and sterilized water. The thermal cycle program for RT-PCR consisted

169 of an initial denaturation at $94^{\circ} \mathrm{C}$ for 1 min followed by 30 cycles of $94^{\circ} \mathrm{C}$ for $30 \mathrm{~s}, 55^{\circ} \mathrm{C}$ for 30

$170 \mathrm{~s}$ and $72^{\circ} \mathrm{C}$ for $45 \mathrm{~s}$, and a final extension at $72^{\circ} \mathrm{C}$ for $5 \mathrm{~min}$. PCR products were analyzed on a

$1712 \%$ agarose gel. Three biological replications (i.e., 3 independent preparations of total RNA),

172 each with three repeated PCR runs, were performed in this analysis.

\section{3. Results}

\subsection{Full-length cDNAs and the deduced amino acid sequences of five AgCht5 genes}

The conceptual translation of the An. gambiae gene model XP_001237469.2 predicts a

1762095 amino acid-long protein with five catalytic domains. However, analysis of the

177 sequences of five cDNA clones (EST clone numbers: 19600449629438 for AgCht5-1,

17819600449653107 for $A g C h t 5-2,19600449656904$ for $A g C h t 5-3$, NAP1-P158-B-06-5 for

$179 A g C h t 5-4$, and 19600449684410 for $A g C h t 5-5)$ that were obtained from the MR4 failed to 
180 provide evidence for a long transcript that bridges genomic sequences encoding adjacent

181 chitinase catalytic domains predicted by this gene model. Instead, we could detect sequences

182 that were presumed to be introns in this gene model at the 5 'ends or 3 'ends of the five

183 full-length chitinase cDNA sequences that we have characterized.

184 The additional sequences at the 3'-ends of these clones (which were not included in the gene model XP_001237469.2) had stop codons and polyadenylation signal sequences and

186 short poly A tails. The additional sequences at the 5'-ends of the full-length cDNA clones

187 included start codons followed by signal peptide coding regions. The sequence data we

188 obtained from full-length cDNA clones are consistent with a gene model that replaces the

189 current XP_001237469.2 with five separate genes encoding five chitinases that differ in their

190 leader peptide as well as catalytic domain sequences. Each of the five cDNA sequences

191 contains a start codon (ATG) and a stop codon (TAA, TAG, or TGA) as well as a poly (A)

192 tail. Except for $A g C h t 5-4$ that lacks a typical polyadenylation signal sequence (AATAAA),

193 all the remaining four cDNAs contain such a signal sequence. These five chitinase-like genes

194 are denoted as AgCht5-1, AgCht5-2, AgCht5-3, AgCht5-4 and AgCht5-5. Their cDNA and

195 deduced amino acid sequences have been deposited in GenBank with the following accession

196 numbers: HQ456129 for AgCht5-1, HQ456130 for AgCht5-2, HQ456131 for AgCht5-3,

197 HQ456132 for AgCht5-4 and HQ456133 for AgCht5-5.

198 Analysis of the genomic organization of the five $A g C h t 5$ genes showed that they form a

199 contiguous cluster of genes in chromosome 2R (Fig. 1A, Table 2). The shortest distance

200 between two of these genes is only $340 \mathrm{bp}$, whereas the longest distance is $2045 \mathrm{bp}$. The

201 percent nucleotide sequence identities among the cDNAs of the five genes range from 52 to 
66\% (Table 3). AgCht5-1 has four introns, $A g C h t 5-2$ and $A g C h t 5-3$ have two introns,

$203 A g C h t 5-4$ has only one intron, whereas $A g C h t 5-5$ has no introns (Fig. 1B).

The five $A g C h t 5$ genes were predicted to encode five chitinase-like proteins with sizes ranging from 409 to 571 amino acid residues (Fig. 1C). The identities of the amino acid sequences among the five full-length deduced proteins range from 38 to $53 \%$ (Table 3). All of the five putative chitinase proteins possess a catalytic domain, but only AgCht5-1 exhibits a chitin-binding domain (Fig. 2). The catalytic domain of each deduced protein is composed of four motif sequences that are conserved among family 18 chitinases. All the five deduced proteins were predicted to possess a signal peptide (Fig. 1C, Fig. 2).

211 To examine whether similar Cht5 gene clusters exist in other mosquito species, we

212 searched for Cht5-related genes in the genome databases of Ae. aegypti and $C$.

213 quinquefasciatus, and identified four AaCht5 and three $C q C h t 5$ genes, respectively. By

214 aligning the deduced amino acid sequences of all of the Cht5s from the three mosquito species and other insect species, we assigned the names AaCht5-1, AaCht5-2, AaCht5-3 and

216 AaCht5-4 for those identified in Ae. aegypti, and CqCht5-1,CqCht5-2 and CqCht3 for those

217 identified in C. quinquefasciatus. Analysis of their domain architectures indicated that all the 218 deduced Cht5 proteins from the three mosquito species have a catalytic domain, but only the

219 first Cht5 protein in each species (i.e., AgCht5-1, AaCht5-1 and CqCht5-1) contains a 220 chitin-binding domain (Fig. 3).

\subsection{Phylogenetic analysis of five deduced AgCht5 protein sequences}

To explore the relationship among the insect Cht5s, a phylogenetic tree was constructed

223 based on the sequences of their catalytic domains. Results showed that all of the insect Cht5s 
fall into two branches supported by a bootstrap value of 100 after 5000 replications (Fig. 4).

The first group represents the mosquito Cht5-1 and all other well characterized insect Cht5s with chitinase activities, whereas the second group represents the remaining mosquito Cht5s. Apparently, AgCht5-1, AaCht5-1 and CqCht5-1 from the three mosquito species are more closely related and might represent mosquito orthologs of insect Cht5 enzymes. In contrast, the mosquito Cht5-2, Cht5-3, Cht5-4 and Cht5-5 are clustered in another branch that may be encoded by genes derived from an ancestral Cht5-1 by gene duplications. Cht5-2s from three mosquito species close together with robust bootsrap value, suggest they are also orthologs.

\subsection{Developmental stage-dependent expression patterns of five AgCht5 genes}

The developmental stage-dependent expression patterns of different $\mathrm{AgCht5}$ transcripts were determined by RT-PCR. The levels of transcripts of the five $\mathrm{AgCht5}$ genes were apparently higher in third- and fourth-instars (Fig. 5A). Four genes including AgCht-1, AgCht5-2, AgCht5-3 and AgCht5-5 were expressed at all developmental stages, whereas $\mathrm{AgCht5-4}$ was expressed mainly in third- and fourth-instar larvae with trace amounts of transcripts detected in the eggs and first-instar larvae. Their detailed expression patterns were further examined in eggs collected at 12, 24, 36, 48 and $60 \mathrm{~h}$ after deposition by blood-fed females (Fig. 5B). High transcript levels were detected in 36-h eggs for all of the five $A g C h t 5 s$. However, no detectable expression was found for $A g C h t 5-2$ in 60 -h eggs and for AgCht5-5 in 12-h eggs. On the other hand, AgCht5-4 was scarcely detected in mature eggs. Similarly, the expression patterns of the five different $\mathrm{AgCht5}$ genes were also examined in pupae collected at 0, 10, 20, 30 and $34 \mathrm{~h}$ after pupation (Fig. 5C). AgCht5-1 and AgCht5-3 were apparently expressed during all the pupal stage and exhibited similar expression patterns, 
whereas $A g C h t 5-2$ and $A g C h t 5-5$ were mainly expressed in 0- and 10-h pupae. However, the expression of $\mathrm{AgCht5-4}$ appeared to gradually increase with pupal development from 0 to 34

h.

\section{Discussion}

Currently, the deduced Cht5 proteins from different insect species are grouped into one clade in the phylogenetic analysis of insect chitinases (Arakane and Muthukrishnan, 2010).

All chitnase 5 proteins possess a typical multiple domain structural organization consisting of a signal peptide, an $\mathrm{N}$-terminal catalytic domain with four conserved motif sequences, KXXXXXGGW, FDGXDLDWEYP, MXYDXXG and GXXXWXXDXD, a S/T-rich linker region and a cysteine-rich chitin-binding domain that conforms to the consensus C- $\left(X_{11}\right)-C-\left(X_{5}\right)-C-\left(X_{9}\right)-C-\left(X_{12}\right)-C-\left(X_{7}\right)-C$ spacing of six cysteines that are predicted to form three disulfide bonds. They are highly expressed in the epidermis, and the transcripts appear just before ecdysis and disappear soon after ecdysis (Kramer, et al., 1993; Zheng et al., 2002). Furthermore, results of RNA interference in $T$. castaneum suggested that Cht5 might be involved in chitin turnover associated with molting (Zhu et al., 2008b). To date, however, only single Cht5 gene has been reported in various insect species.

From our genome-wide searching of the An. gambiae genome, we putatively identified a chitinase 5 gene $(A g C h t 5)$ (accession no: XP_001237469.2) that has been annotated in

264 Vectorbase to encode a large protein with five different catalytic domains. However, our careful studies unexpectedly revealed different gene expression patterns when we used unique primer sets designed to amplify cDNAs from specific regions of $A g C h t 5$. This finding

267 prompted us to hypothesize that $A g C h t 5$ actually is a gene cluster consisting of multiple 
genes and the different catalytic domains might be encoded by different genes. To address this question, we utilized the cDNA sequence of each domain of AgCht5 to search the An. gambiae EST database to determine whether separate transcripts corresponding to the five catalytic domains existed in the EST database. As expected, we obtained five full-length cDNA sequences, each corresponding to only one of the five catalytic domains. No evidence for an EST with sequences from two or more adjoining Cht5 coding regions was obtained.

274 Each of these five full-length cDNAs in the EST database apparently encode a chitinase containing only one catalytic domain with all four conserved motif sequences expected of chitinases (Fig. 2). As supported by the different expression patterns of these genes during different developmental stages of the mosquito, we conclude that the previously reported $A n$. gambiae Cht5 gene model actually represents a unique gene cluster consisting of five different chitinase or chitinase-like genes. To our knowledge, this is the first report on a multiple-member Cht5 gene cluster in insects.

Our further studies confirmed a similar clustering of $C h t 5$ genes in other mosquito species. Based on our genome search, we identified four Cht5-like genes in Ae. aegypti and three in $C$. quinquefasciatus. By aligning these mosquito Cht5 proteins with other known insect Cht5 proteins, we found that only one Cht5 catalytic domain (AgCht5-1, AaCht5-1 or

285 CqCht5-1) from each mosquito species was clustered with other domains from known insect

286 Cht5 proteins with a bootstrap value of 100 (Fig. 4), whereas the remaining Cht5s from these 287 three mosquito species were grouped into a different cluster. These results suggest that AgCht5-1, AaCht5-1, CqCht5-1 and all other known insect Cht5s are orthologous genes, whereas the remaining four $A g C h t 5$, three $A a C h t 5$ and two $C q C h t 5$ genes are paralogous to 
clustered together with high bootstrap value, and represented similar domain architecture

292 (Fig.3), suggesting that Cht5-2s from three mosquito species may be also orthologous genes.

293 The absence of paralogs of Cht5 genes in D. melanogaster and T. castaneum suggests that

294 amplification of this subgroup of chitinase genes is of recent origin.

295 The catalytic and chitin-binding domains are two important structural components of

296 chitinases. Sequence motif analysis showed that all mosquito Cht5 proteins possess a

297 catalytic domain, but only three of the proteins, AgCht5-1, AaCht5-1 and CqCht5-1, contain

298 the signature sequence DWEYP within conserved region II, which is known to be located in

299 or near the catalytic site of the enzyme. The third residue $\mathrm{E}$ is crucial for a chitinase being

300 catalytically active because it probably serves as a proton donor in the catalytic mechanism

301 (Watanabe et al., 1994). Thus, AgCht5-1, AaCht5-1 and CqCht5-1 are predicted to be catalytically active, which are similar with all the other known insect Cht5s. In contrast, all of the other predicted Cht5-like proteins from the three mosquito species are likely to be catalytically inactive because the E residue is replaced by $\mathrm{L}$ in these proteins. These proteins may have carbohydrate-binding capability. Domain analysis revealed that only AgCht5-1,

AaCht5-1 and CqCht5-1 possess a chitin-binding domain. The function of chitin-binding

307 domain is presumably to anchor the enzyme tightly onto the large insoluble polymeric

308 substrate, thereby facilitating the hydrolytic process (Arakane et al., 2003, Boot et al., 2001).

309 Our analysis of the genomic organization of the $A g C h t 5$ cluster suggests that this gene

310 cluster could be evolved from gene duplications. Gene duplication events are generally

311 considered to be essential in the evolution of gene families, which facilitate the generation of 
312 new genes with new functions. Gene duplication can occur via three major mechanisms:

313 segmental duplication (of the whole genome, of one to a few chromosomes or of large parts

314 of a chromosome), tandem duplications (of one to a few adjacent genes), and retroposition or

315 other transposition events (Kong et al., 2007). Among these, tandem and segmental

316 duplication events contribute mostly to the generation of new members in nuclear gene

317 families. Tandem duplicates are copies of a nearby gene that are within short intron distances

318 of each other and may harbor some interesting biology. Gene expansion by tandem

319 duplication is common in cytochrome P450 gene evolution (Ai et al., 2010; Baldwin et al.,

320 2009). Five AgCht5 genes are clustered together in chromosome $2 \mathrm{R}$ with no other

321 intervening genes. The minimum distance is $340 \mathrm{bp}$ between two chitinase ORFs and the

322 maximum is $2045 \mathrm{bp}$, suggesting that these genes may be derived from tandem duplications.

323 Gene duplication and loss according to a birth-and-death model of evolution is a feature of

324 the evolutionary history of the family 18 (GH18) of chitinases (Funkhouser et al., 2007).

325 Based on above information, we proposed that AgCht5 gene cluster may evolve primarily

326 from tandem duplication.

327

Because the transcripts of all the five $\mathrm{AgCht5}$ genes can be detected at various developmental stages in An. gambiae (Fig. 5), these genes are transcribed and appear to be independently regulated. However, the deduced AgCht5-2, AgCht5-3, AgCht5-4 and

330 AgCht5-5 proteins lack an essential catalytic glutamic acid (Watanabe et al., 1994, Lu et al.,

331 2002, Zhang et al., 2002, Zhu et al., 2008a). Therefore, they are presumed to act as

332 carbohydrate-binding proteins or lectins rather than as enzymes because they may not have any catalytic activity (Goormachtig et al., 2001). Nevertheless, it should be pointed out that a 
334 chitinase-like protein without catalytic activity may still play an important role in insect

335 development. For example, insect imaginal disc growth factors (IDGFs) are chitinase-like

336 proteins that are structurally related to chitinases but do not possess enzymatic activity.

337 However, TcIDGF4 identified in T. castaneum might be involved in cell proliferation and

338 contributed to adults ecdysis (Zhu et al., 2008b). Further studies will be necessary to

339 elucidate the biological function of each of the five duplicated Cht5 genes in An. gambiae.

340 


\section{Acknowledgements}

342 Authors thank Sharon R. Starkey for maintaining mosquito colonies. This research was

343 supported in part by Kansas Agricultural Experiment Station and K-State Arthropod

344 Genomics Center funded by K-State Targeted Excellence program at Kansas State University.

345 This manuscript is contribution No. 11-194-J from the Kansas Agricultural Experiment

346 Station, Kansas State University, Manhattan, Kansas, USA. The Anopheles gambiae voucher

347 specimens (voucher No. 211) are located in the Kansas State University Museum of

348 Entomological and Prairie Arthropod Research, Manhattan, Kansas.

349

350

351

352

353

354

355

356

357

358 


\section{References}

360 Ahmad, T., Rajagopal, R., Bhatnagar, R.K., 2003. Molecular characterization of chitinase from polyphagous pest Helicoverpa armigera. Biochem. Biophys. Res. Comm. 310, 188-195.

Ai, J., Yu, Q., Cheng, T., Dai, F., Zhang, X., Zhu, Y., Xiang Z., 2010. Characterization of multiple CYP9A genes in the silkworm Bombyx mori. Mol. Biol. Rep. 37, 1657-1664.

Arakane, Y., Muthukrishnan, S., 2010. Insect chitinase and chitinase-like proteins. Cell. Mol. Life Sci. 67, 201-216.

Arakane, Y., Zhu, Q., Matsumiya, M., Muthukrishnan, S., Kramer, K.J., 2003. Properties of catalytic, linker and chitin-binding domains of insect chitinase. Insect Biochem. Mol. Biol. 33, 631-648.

Baldwin, W.S., Marko, P.B., Nelson D.R., 2009. The cytochrome P450 (CYP) gene superfamily in Daphnia pulex. BMC Genomics 10, 169.

Bolognesi, R., Arakane, Y., Muthukrishnan, S., Kramer, K.J., Terra, W.R., Ferreira, C., 2005. Sequences of cDNAs and expression of genes encoding chitin synthase and chitinase in the midgut of Spodoptera frugiperda. Insect Biochem. Mol. Biol. 35, 1249-1259.

Boot, R.G., Blommaart, E.F., Swart, E., Ghauharali-van der Vlugt, K., Bijl, N., Moe, C., Place, A., Aerts, J.M., 2001. Identification of a novel acidic mammalian chitinase distinct from chitotriosidase. J. Biol. Chem. 276, 6770-6778.

Filho, B. P.D., Lemos, F. J.A., Secundino, N. F.C., Pascoa V., Pereira, S. T., Pimenta, P. F.P., 2002. Presence of chitinase and beta-N-acetylglucosaminidase in the Aedes aegypti. a 
chitinolytic system involving peritrophic matrix formation and degradation. Insect Biochem. Mol. Biol. 32, 1723-1729.

382

Fitches, E., Wilkinson, H., Bell, H., Bown, D.P., Gatehouse, J.A., Edwards, J.P., 2004. Cloning, expression and functional characterization of chitinase from larvae of tomato moth (Lacanobia oleracea): a demonstration of the insecticidal activity of insect chitinase. Insect Biochem. Mol. Biol. 34, 1037-1050.

Funkhouser, J.D., Aronson, N.N. Jr., 2007. Chitinase family GH18: Evolutionary insights from the genomic history of a diverse protein family. BMC Evol. Biol. 7, 96.

Genta, F. A., Blanes, L., Cristofoletti, P.T., do Lago, C. L., Terra W. R. and Ferreira, C., 2006. Purification, characterization and molecular cloning of the major chitinase from Tenebrio molitor larval midgut. Insect Biochem. Mol. Biol. 36, 789-800.

Goormachtig, S., Van de Velde, W., Lievens, S., Verplancke, C., Herman, S., De Keyser, A., Holsters, M., 2001. Srchi24, a chitinase homolog lacking an essential glutamic acid residue for hydrolytic activity, is induced during nodule development on Sesbania rostrata. Plant Physiol. 127, 78-89.

Gopalakrishnan, B., Muthukrishnan, S., Kramer, K. J., 1995. Baculovirus-mediated expression of a Manduca sexta chitinase gene: Properties of the recombinant protein. Insect Biochem. Mol. Biol. 25, 255-265.

Hirose, T., Sunazuka, T., Omura, S., 2010. Recent development of two chitinase inhibitors, Argin and Argadin, produced by soil microorganisms. Proc. Jpn. Acad., Series B 86, 85-102. 
401

402

403

404

405

406

407

408

409

410

411

412

413

414

415

416

417

418

419

420

421

422

Khajuria, C., Buschman, L.L., Chen,M.-S., Muthukrishnan, S., Zhu, K.Y., 2010. A gut-specific chitinase gene essential for regulation of chitin content of peritrophic matrix and growth of Ostrinia nubilalis larvae. Insect Biochem. Mol. Biol. 40, 621-629.

Kim, M.G., Shin, S.W., Bae, K.S., Kim, S.C., Park, H.Y., 1998. Molecular cloning of chitinase cDNAs from the silkworm, Bombyx mori and the fall webworm, Hyphantria cunea. Insect Biochem. Mol. Bio. 28, 163-171.

Kong, H., Landherr, L.L., Frohlich, M.W., Leebens-Mack J., Ma, H. and dePamphilis, C.W., 2007. Patterns of gene duplication in the plant SKP1 gene family angiosperms: Evidence for multiple mechanisms of rapid gene birth. Plant J. 50, 873-885.

Kramer, K.J., Corpuz, L., Choi, H.K., Muthukrishnan, S., 1993. Sequence of a cDNA and expression of the gene encoding epidermal and gut chitinases of Manduca sexta. Insect Biochem. Mol. Biol. 23, 691-701.

Krishnan, A., Nair, P.N., Jones, D., 1994. Isolation, cloning, and characterization of new chitinase stored in active form in chitin lined venom reservoir. J. Biol. Chem. 269, 20971-20976.

Lu, Y., Zen, K.C., Muthukrishnan, S., Kramer, K.J., 2002. Site-directed mutagenesis and functional analysis of active site acidic amino acid residues D142, D144 and E146 in Manduca sexta (tobacco hornworm) chitinase. Insect Biochem. Mol. Biol. 32, 1369-1382.

Reese, T.A., Liang, H.E., Tager, A.M., Luster, A.D., Rooijen, V.N., Voehringer, D., Locksley, R.M., 2007. Chitin induces accumulation in tissue of innate immune cells associated with allergy. Nature Let. 447, 92-96. 
423

424

425

426

427

428

430

431

432

433

434

435

436

437

438

439

440

441

442

Reynolds, S. E. and Samuels, R. I., 1996. Physiology and biochemistry of insect moulting fluid. Adv. Insect Physiol. 26, 158-232.

Royer, V., Fraichard, S., Bouhin, H., 2002. A novel putative insect chitinase with multiple catalytic domains: Hormonal regulation during metamorphosis. Biochem. J. 366, 921-928.

Schultz, J., Milpetz, F., Bork, P., Ponting, C.P., 1998. SMART, a simple modular architecture research tool: identification of signaling domains. Proc. Natl. Acad. Sci. U.S.A. 95, $5857-5864$.

Shen, Z., Jacobs-Lorena, M., 1997. Characterization of a novel gut specific chitinase gene from the human malaria vector Anopheles gambiae. J. Biol. Chem. 272, 28895-28900.

Shinoda, T., Kobayashi, J., Matsui, M., Chinzei, Y., 2001. Cloning and functional expression of a chitinase cDNA from the common cutworm, Spodoptera litura, using a recombinant baculovirus lacking the virus-encoded chitinase gene. Insect Biochem. Mol. Biol. 1, 521-532.

Shuhui, L., Mok, Y.K., Wong ,W.S.F., 2009. Role of Mammalian Chitinases in Asthma. Int. Arch. Allergy Immunol. 149, 369-377.

Spindler-Barth, M., Blattner, R., Vorgias, C.E., Spindler, K.D., 1998. Inhibition of two family 18 chitinases by various allosamidin derivatives. Pestic. Sci. 52, 47-52.

Tamura, K., Dudley, J., Nei, M., Kumar, S., 2007. MEGA4: Molecular Evolutionary Genetics Analysis (MEGA) software version 4.0. Mol. Biol. Evol. 24, 1596-1599. 
Watanabe, T., Uchida, M., Kobori, K., Tanaka, H., 1994. Site-directed mutagenesis of the Asp-197 and Asp-202 residues in chitinase A1 of Bacillus circulans WL-12. Biosci. Biotechnol. Biochem. 58, 2283-2285.

Zhang, H., Huang, X., Fukamizo, T., Muthukrishnan, S., Kramer, K.J., 2002. Site-directed mutagenesis and functional analysis of an active site tryptophan of insect chitinase. Insect Biochem. Mol. Biol. 32, 1477-1488.

Zhang, J.Z., Zhu, K.Y., 2006. Characterization of a chitin synthase cDNA and its increased mRNA level associated with decreased chitin synthesis in Anopheles quadrimaculatus exposed to diflubenzuron. Insect Biochem. Mol. Bio. 36, 712-725.

Zheng, Y., Zheng, S., Cheng, X., Ladd, T., Lingohr, E.J., Krell, P.J., Arif, B.M., Retnakaran, A., Feng, Q., 2002. A molt-associated chitinase cDNA from the spruce budworm, Choristoneura fumiferana. Insect Biochem. Mol. Biol. 32, 1813-1823.

Zheng, Y.P., Retnakaran, A., Krell, P.J., Arif, B.M., Primavera, M., Feng Q.L., 2003. Temporal, spatial and induced expression of chitinase in the spruce budworm, Choristoneura fumiferana. J. Insect Physiol. 49, 241-247.

Zhu, Q., Arakane Y, Banerjee, D., Beeman, RW, Kramer, K.J., Muthukrishnan, S., 2008a. Domain organization and phylogenetic analysis of the chitinase like family of proteins in three species of insects. Insect Biochem. Mol. Biol. 38, 452-466.

Zhu, Q., Arakane, Y., Beeman, R.W., Kramer, K.J., Muthukrishnan, S., 2008b. Functional specialization among insect chitinase family genes revealed by RNA interference. Proc. Natl. Acad. Sci. U.S.A. 105, 6650-6655. 
464 Zhu, Q., Deng, Y., Vanka, P., Brown, S.J., Muthukrishnan, S., Kramer, K.J., 2004.

465 Computational identification of novel chitinase-like proteins in the Drosophila

466 melanogaster genome. Bioinformatics 20, 161-169.

467

468

469

470

471

472

473

474

475

476

477

478

479

480

481

482

483

484

485

486

487

488

489

490

491

492

493

494

495

496

497

498

499

500

501

502 


\section{Figure legends:}

505 Fig. 1. Genome structure, exon/intron organizations and protein domain architectures of

506 five $A g C h t 5$ genes. A) Genome structure of $A g C h t 5-1, A g C h t 5-2, A g C h t 5-3, A g C h t 5-4$ and

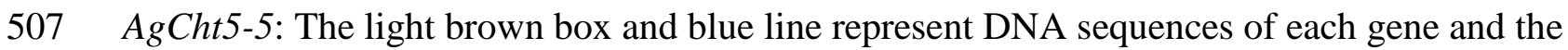
508 linker region, respectively, between the ORF's. The length of linker region is marked under 509 the blue line and by a yellow triangle. B) Exon and intron organizations: Exons are shown by

510 green boxes whereas introns are shown by pink lines. C) Domain architectures of predicted

511 AgCht5 proteins: Predicted signal peptide, catalytic domain and chitin-binding domain are

512 boxed in yellow, gray and purple, respectively, whereas linker regions are shown by blue 513 lines.

515 Fig. 2. Multiple alignments of deduced amino acid sequences of AgCht5's. Signal peptide, 516 catalytic domain and chitin-binding domain are highlighted in orange, light blue and green,

517 respectively, on the top of aligned sequences. The four conserved motif sequences are boxed

518 in blue and denoted as CR1, CR2, CR3 and CR4. Fully conserved amino acid sequences are 519 shaded in black.

521 Fig. 3. Comparative analysis of domain architecture of Cht5s from three mosquito species.

522 The red line, blue triangle and light green hexagon represent the signal peptide, catalytic

523 domain and chitin-binding domain, respectively. 
Fig. 4. Phylogenetic analysis of catalytic domain sequences of putative chitinase 5 proteins from different species including Aedes aegypti (Aa, XP_001656234.1 for AaCht5-1, XP_001656233.1 for AaCht5-2, XP_001656232.1 for AaCht5-3, and XP_001656231.1 for AaCht5-4), Anopheles gambiae (Ag, HQ456129 for AgCht5-1, HQ456130 for AgCht5-2, HQ456131 for AgCht5-3, HQ456132 for AgCht5-4, and HQ456133 for AgCht5-5), Bombyx mandarina (Bma, AAG48700.1), Bombyx mori (Bmo, AAB47538), Choristoneura fumiferana (Cf, AAM43792), Culex quinquefasciatus (Cq, XP_001863384.1 for CqCht5-1, XP_001863385.1 for CqCht5-2, and XP_001863386.1 for CqCht5-3,) Drosophila melanogaster (Dm, CG9307), Helicoverpa armigera (Ha, AAQ91786), Hyphantria cunea (Hc, AAB47537), Lacanobia oleracea (Lo, CAF05663), Manduca sexta (Ms, P36362), Spodoptera frugiperda (Sf, AAS18266), Spodoptera litura (S1, AB032107), and Tribolium castaneum (Tc, AY675073 ). The phylogenetic tree was constructed using Mega 4 software

537 (Tamura et al., 2007). Bootstrap values are obtained by the neighbor-joining method using 5385000 replications. Bootstrap values are indicated only when greater than $40 \%$.

Fig. 5. The expression patterns of five $A g C h t 5$ genes in Anopheles gambiae as evaluated using RT-PCR. A) Gene expression patterns in eggs (EG), first- (L1), second- (L2), third-

542 (L3) fourth- (L4) and fifth-instar larvae (L5); and adults (AD). B) Gene expression patterns in 543 12-, 24-, 36-, 48- and 60 h-old eggs as shown by EG12, EG24, EG36, EG48, and EG60,

544 respectively. C) Gene expression patterns in 0-, 10-, 20-, 30- and 34-h-old eggs as shown by 545 PU00, PU10, PU20, PU30 and PU34, respectively. AgRps3 was used as reference gene for 546 RT-PCR analysis. 
547 Table 1. Sequences of PCR primers used in expression analyses of five AgCht5 genes and 548 3'RACE primers used in amplifications of full-length cDNAs for AgCht5-1 and AgCht5-4

549 genes.

550

\begin{tabular}{|c|c|c|}
\hline Primer name & Sequence (5'-3') & $\begin{array}{l}\text { Product size } \\
\text { (base pairs) }\end{array}$ \\
\hline$A g C h t 5-1-\mathrm{F}$ & TTCCGGCTACAAGGACTTTG & 188 \\
\hline AgCht5-1-R & TCGGGCTTTCGATCAGTTTC & \\
\hline$A g C h t 5-2-\mathrm{F}$ & ACGATAAGGACAACTTTGTCTATC & 152 \\
\hline AgCht5-2-R & GTCAGCACTCTCGCACAG & \\
\hline AgCht5-3-F & GCTGTGTGAAATGCTGAAGG & 166 \\
\hline AgCht5-3-R & TGCGTATATGCCACCCAATC & \\
\hline AgCht5-4-F & TTCGCCAACCTGAAGAAGAC & 146 \\
\hline AgCht5-4-R & TGGAGGAACTCAATCACACTG & \\
\hline AgCht5-5-F & TTCATCGGCAGCGTGATC & 197 \\
\hline AgCht5-5-R & TCGACCGGCACCTGTATC & \\
\hline $\begin{array}{l}\text { AgCht5-1- } \\
\text { 3'RACE-N1 }\end{array}$ & ACGAGGACGAACGGTCGCTCCAGCAC & 900 \\
\hline $\begin{array}{l}\text { AgCht5-1- } \\
\text { 3'RACE-N2 }\end{array}$ & TGGACGATTTCCACGGTCTTTGCGGGCCG & 780 \\
\hline $\begin{array}{l}\text { AgCht5-4- } \\
\text { 3'RACE-N1 }\end{array}$ & ACTAGCAAACAGCGAGGAGCATGGACTG & 680 \\
\hline $\begin{array}{l}\text { AgCht5-4- } \\
\text { 3'RACE-N2 }\end{array}$ & ACCGAAATGCAGCAGTCCGGCTGGGAG & 520 \\
\hline
\end{tabular}



in the NCBI database and genome location of each $A g C h t 5$ gene from An. gambiae.

555

\begin{tabular}{llllll}
\hline Gene & $\begin{array}{l}\text { Amino acid } \\
\text { residue }\end{array}$ & $\begin{array}{l}\text { Catalytic } \\
\text { domain }\end{array}$ & $\begin{array}{l}\text { Chitin-binding } \\
\text { domain }\end{array}$ & $\begin{array}{l}\text { Availability of } \\
\text { EST* }\end{array}$ & Genome location \\
\hline AgCht5-1 & 571 & Yes & Yes & Yes & chr2R:21,584,333-21,587,318 \\
AgCht5-2 & 412 & Yes & No & Yes & chr2R:21,582,374-21,583,826 \\
AgCht5-3 & 413 & Yes & No & Yes & chr2R:21,578,829-21,580,211 \\
AgCht5-4 & 409 & Yes & No & Yes & chr2R:21,576,773-21,578,085 \\
$A g C h t 5-5$ & 446 & Yes & No & Yes & chr2R:21,573,544-21,574,884 \\
\hline
\end{tabular}

556

$557 *$ Based on the Anopheles gambiae EST database from NCBI 
558 Table 3. Percent identities of amino acid residues (nucleotides) among the ORF's of five

559 AgCht5 chitinase-like genes from An. gambiae.

560

\begin{tabular}{c|c|c|cc}
\hline & AgCht5-2 & AgCht5-3 & AgCht5-4 & AgCht5-5 \\
\hline AgCht5-1 & $43(58)$ & $38(52)$ & $40(55)$ & $41(57)$ \\
\hline AgCht5-2 & & $51(59)$ & $53(63)$ & $50(66)$ \\
\hline AgCht5-3 & & & $47(57)$ & $46(58)$ \\
\hline AgCht5-4 & & & & $45(58)$ \\
\hline
\end{tabular}

561

562

563

564

565

566

567

568

569

570

571

572

573

574

575 
Fig. 1

577

578

579

580 A

581

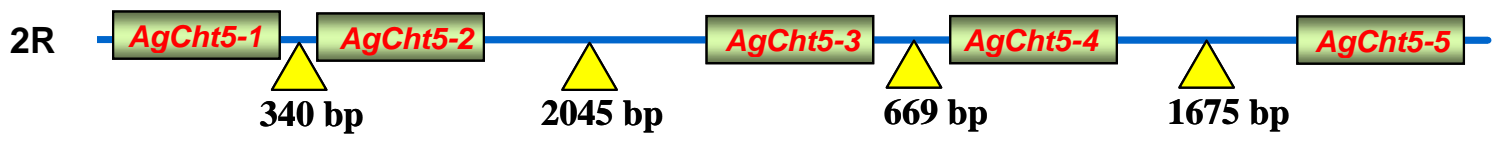

582

583

B

584

585

586

587

588

589

590

591

592

593

594

595

596

597

.

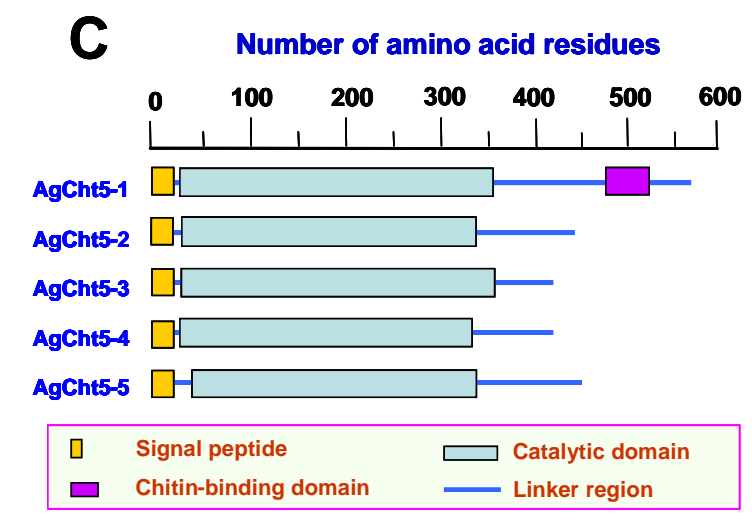

Number of nucleotides (kb)

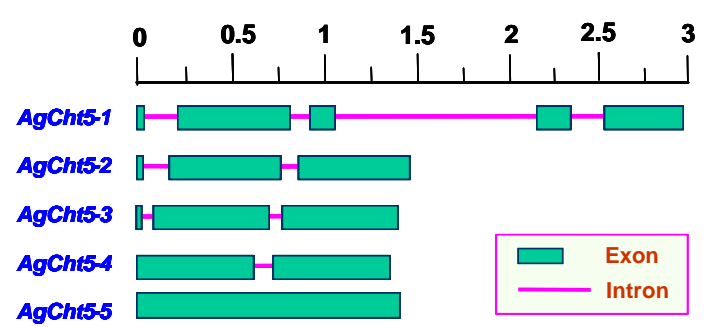

A 
AgCht5-3 AgCht5-5 AgCht5-2

AgCht5-4

AgCht5-1

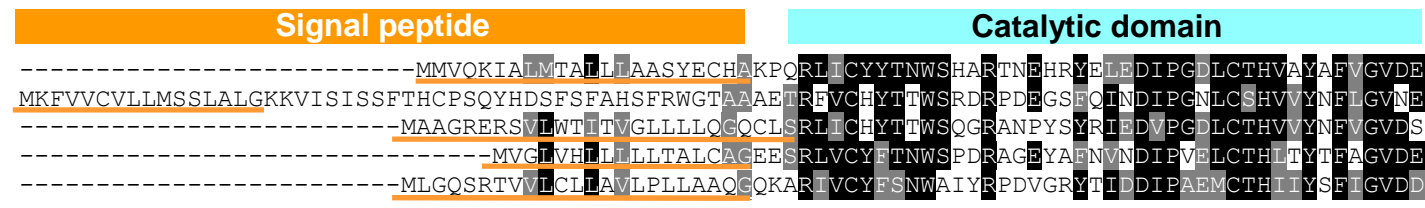

603

AgCht5-3 AgCht5-5

AgCht5-2

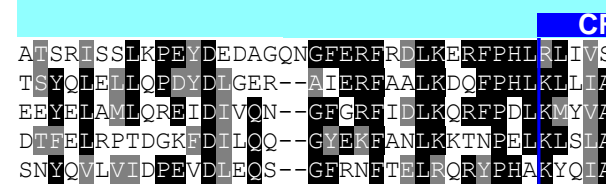

AgCht5-1

605

AgCht5-3 AgCht5-5 AgCht5-2

AgCht5-4
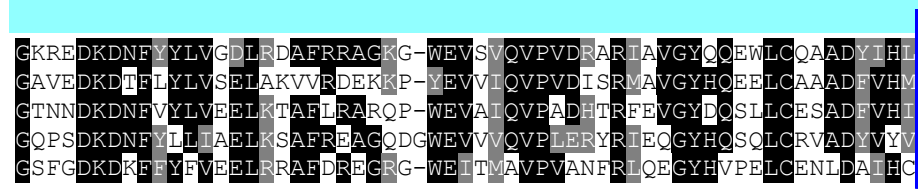

CR3

GYDLRGPWTGYADVHSVLRRR--SHDVHYFAT YYDLRGSWT GFADVHSPMTDR--PHDQGIYKG AgCht5-1

607

AgCht5-3 AgCht5-5

AgCht5-2

AgCht5-4

AgCht 5-1

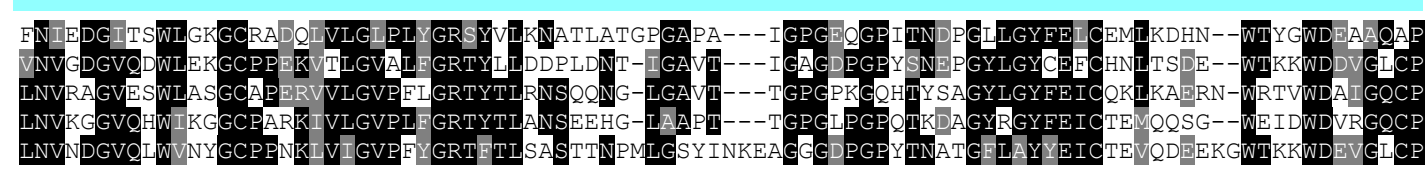

609

AgCht5-3 AgCht5-5 AgCht 5-2

610

Cht5-

AgCht5-1

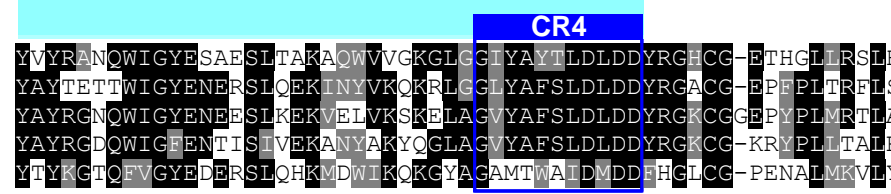

611

AgCht5-3

AgCht5-5

612

AgCht 5-2

AgCht5-4

TTARPATTTTTYKPRPTTTVAPTTRTTTARRTTTTRKPTTILPPDTDSEEDREEPAMPPAAPEREDESEIDCSGYKDFVPSVDCTKYYRCVH

613

AgCht5-3

AgCht5-5

614

AgCht $5-2$

AgCht5-

AgCht5-1

615

616

617

618

619 
$620 \quad$ Fig. 3

621

622

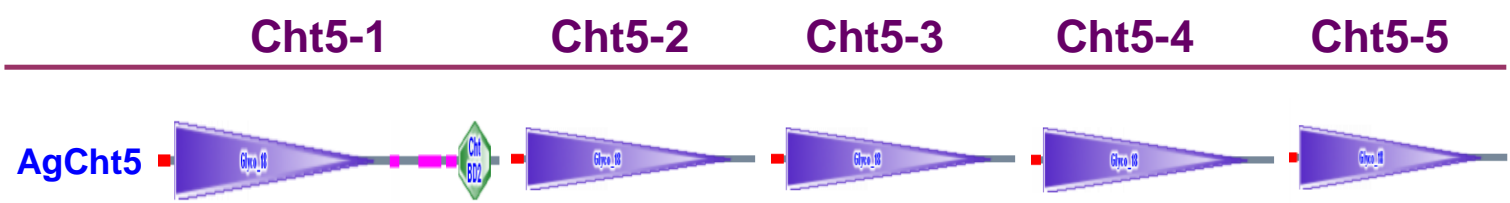

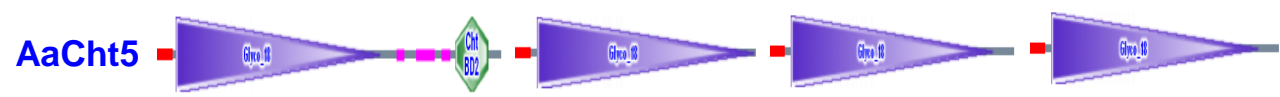

CqCht5

623

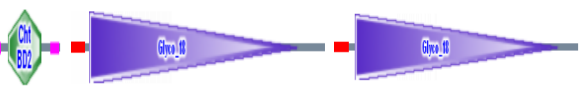

624

625

626

627

628

629

630

631

632

633

634

635

636

637 
$638 \quad$ Fig. 4

639

640

641

642

643

644

645

646

647

648

649

650

651

652

653

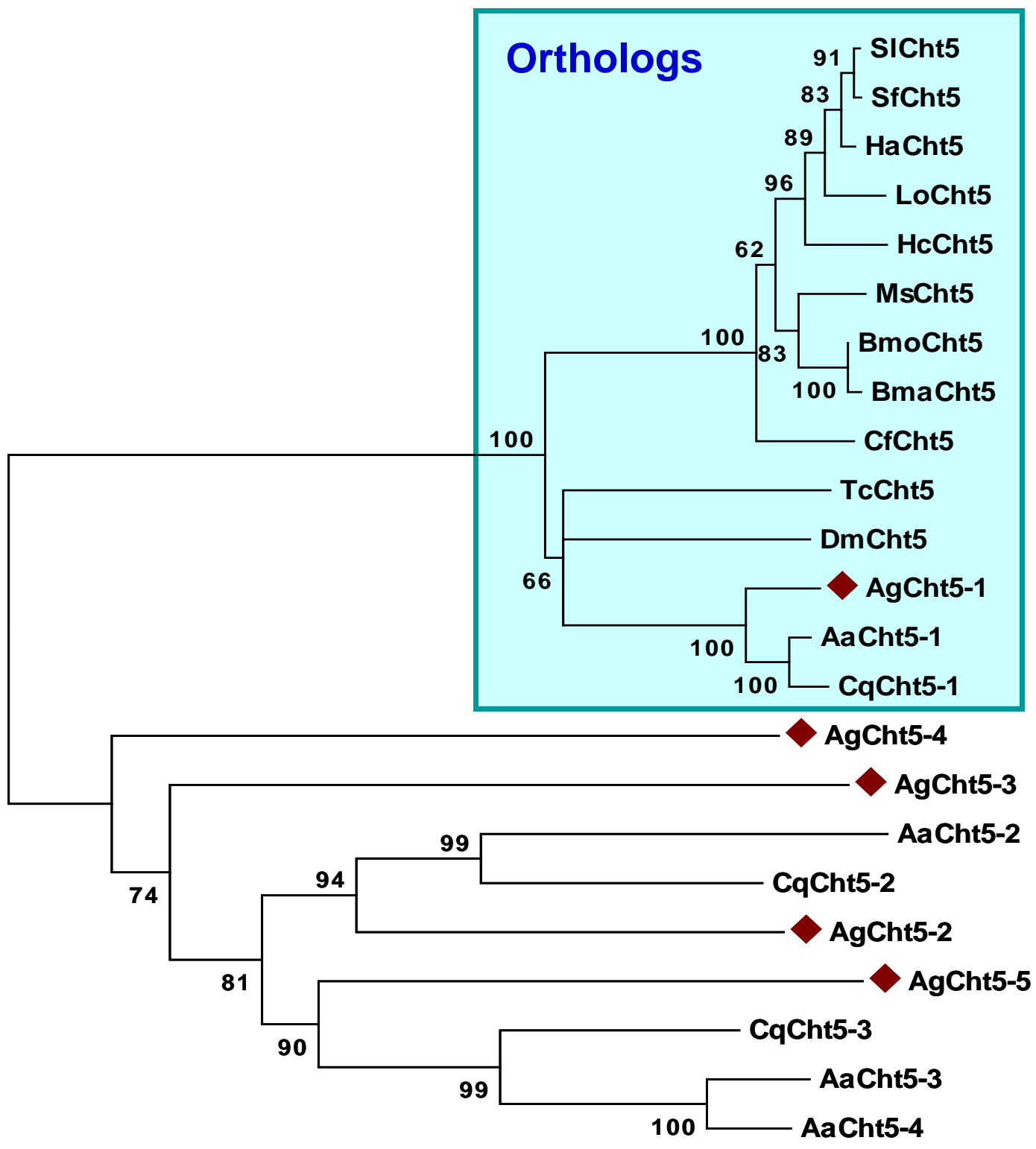

654

655

0.1

656

657

658

659 
660 Fig. 5

661

662

A $\quad$\begin{tabular}{lllllll} 
EG L1 & L2 & L3 & L4 & PU & AD \\
\hline
\end{tabular}

663

AgCht5-1

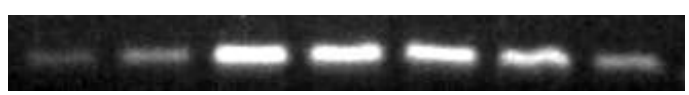

664

AgCht5-2

AgCht5-3

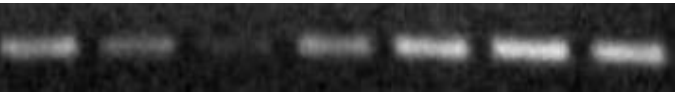

665

AgCht5-4

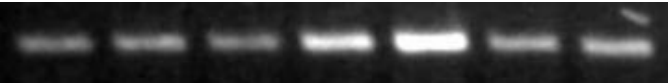

AgCht5-5

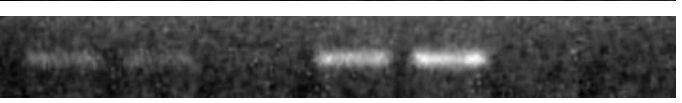

666

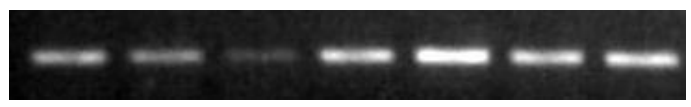

667

668

B

669

AgCht5-1

AgCht5-2

670

AgCht5-3

671

AgCht5-4

672

AgCht5-5

AgRps3
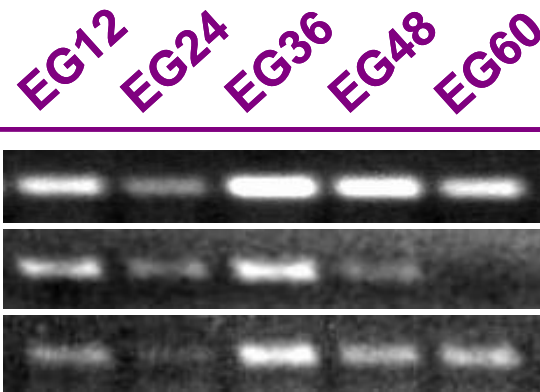

673
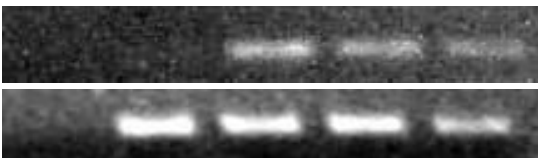

AgRps3

674

675

C

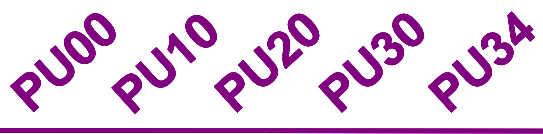

676

AgCht5-1

AgCht5-2

677

AgCht5-3

AgCht5-4

AgCht5-5

679

AgRps3

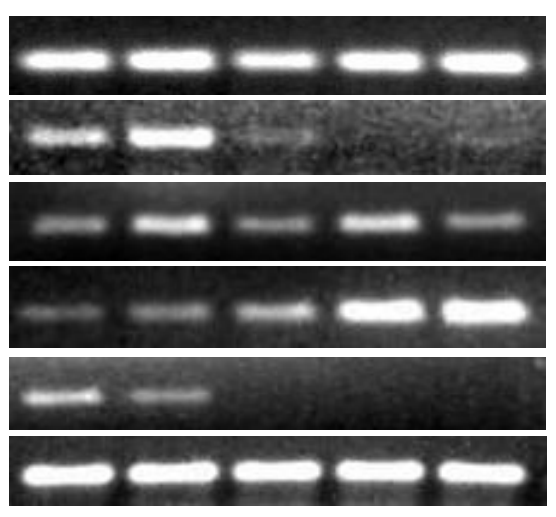

680

681 